\title{
Potential role of serum mesothelin in predicting survival of patients with malignant pleural mesothelioma
}

\author{
VINCENZO FONTANA ${ }^{1}$, MARIA PIA PISTILLO ${ }^{2}$, ANTONELLA VIGANI $^{3}$, PIER ALDO CANESSA ${ }^{4}$, \\ GIOVANNI BERISSO $^{5}$, UGO GIANNONI ${ }^{6}$, PAOLA FERRO ${ }^{7}$, MARIA CRISTIANA FRANCESCHINI $^{7}$, \\ ROBERTA CAROSIO $^{2}$, MARIKA TONARELLI $^{7}$, PAOLO DESSANTI $^{7}$ and SILVIO RONCELLA ${ }^{7}$ \\ ${ }^{1}$ Clinical Epidemiology Unit and ${ }^{2}$ Tumor Epigenetics Unit, IRCCS Ospedale Policlinico San Martino, I-16132 Genova; \\ Departments of ${ }^{3}$ Oncology, ${ }^{4}$ Pneumology, ${ }^{5}$ Medicine, ${ }^{6}$ Radiodiagnosis, and ${ }^{7}$ Histopathology and Cytopathology, \\ Azienda Sanitaria Locale 5, I-19124 La Spezia, Italy
}

Received April 25, 2020; Accepted October 5, 2020

DOI: $10.3892 / 01.2020 .12389$

\begin{abstract}
Malignant pleural mesothelioma (MPM) is an aggressive tumor with poor survival rates. Therefore, it is essential to have effective biological markers predicting the course of the disease and prognosis. The aim of the present study was to highlight the prognostic significance of serum soluble mesothelin-related protein (Se-SMRP) in patients with MPM at diagnosis. Se-SMRP was determined in 60 patients using an ELISA commercial kit. Se-SMRP levels were subdivided into three tertile-based categories and in each category overall survival (OS) indexes were determined using the Kaplan-Meier and Cox regression analyses. The association between Se-SMRP levels and OS was also assessed by restricted cubic spline (RCS) analysis. No notable differences in the Kaplan-Meier probabilities were identified across the Se-SMRP categories (<0.66 nM, 0.66-1.46 nM, >1.46 nM) although an upward trend in death rate ratios (RR) was pointed out by comparing the higher $(R R=1.95)$ and intermediate $(R R=1.86)$ categories with the lower category $(R R=1.00)$. In addition, such an increasing tendency, particularly when the biomarker exceeded $1.0 \mathrm{nM}$, was confirmed by an RCS function of Se-SMPR levels fitted to survival data using the Cox regression equation. The present study provided evidence in favor of a prognostic value of Se-SMRP in patients with MPM.
\end{abstract}

\section{Introduction}

Malignant pleural mesothelioma (MPM) is a highly aggressive tumor of pleural mesothelial cell associated with asbestos exposure $(1,2)$ with a long latency period $(\sim 30-40$ years $)$, an incidence

Correspondence to: Dr Silvio Roncella, Department of Histopathology and Cytopathology, Azienda Sanitaria Locale 5, 2 Via Mario Asso, I-19124 La Spezia, Italy

E-mail: roncy88@alice.it

Key words: mesothelioma, serum, mesothelin, survival, prognosis expected to increase worldwide prior to $2025(3,4)$ and a poor life expectancy (median survival time of $\sim 12$ months) (2).

MPM can be divided into three major histological subtypes: Epithelioid, accounting for $\sim 60 \%$ of MPM and characterized by a longer survival time (range, 12-27 months); sarcomatoid, representing $20 \%$ of all MPM and showing a shorter survival time (range, 7-18 months); biphasic (a combination of epithelioid and sarcomatoid histology), including the residual $20 \%$ with an intermediate survival time (range, 8-21 months) (5).

Determination of prognostic markers of MPM at diagnosis may aid in managing the patient in the therapeutic and follow-up settings $(6,7)$.

For determining MPM prognosis, the scoring systems of the Cancer and Leukemia Group B (CALGB) and the European Organization for Research and Treatment of Cancer (EORTC) are the most useful among those currently available. These systems identify a low performance status score, a non-epithelioid histology, weight loss, male sex, leukocytosis and low hemoglobin as factors associated with a poor prognosis (6-8).

However, performance status and histology are considered, by clinicians, to have the main prognostic role (6-8).

Despite these scoring systems, there is a requirement for novel, highly sensitive and specific biological markers for MPM (9).

Genetic markers with diagnostic or prognostic value are currently under investigation in MPM tumor tissues. Chromosomal alterations, DNA mutations, gene expression profile and the deregulation of microRNA are very promising markers (10-12). Moreover, interesting results derive also from studies on circulating tumor cells, free DNA and microRNA present in body fluids (6).

In recent years, other biomarkers have been proposed in body fluids, including osteopontin, fibulin-3 and vascular endothelial growth factor evaluated in plasma, serum and pleural effusion, in addition to hyaluronic acid evaluated in serum and pleural effusion and the high mobility group box 1 detected only in serum $(6,13,14)$. They showed a low prognostic significance.

However, due to the small number of samples and the heterogeneity of patients analyzed in the individual studies, 
the clinical application of all the markers mentioned above, in MPM, requires further confirmation $(6,13,14)$.

The soluble mesothelin-related protein (SMRP) serves an important role in the diagnosis of MPM as its levels in pleural effusion (PE-SMRP) and in serum (Se-SMRP) have been found to be significantly increased and associated with tumor size $(15,16)$. Therefore, the US Food and Drug Administration approved the SMRP measurement as a routine test (Mesomark ${ }^{\mathrm{TM}}$ ELISA) to aid in diagnosing and monitoring of patients with epithelioid or biphasic MPM.

Although, in MPM, Se-SMRP shows a lower diagnostic performance with respect to PE-SMRP (15), its levels have been used to distinguish patients with MPM from individuals with benign pleural diseases (17) and from patients with pleural metastases of different types (18). However, the diagnostic performance of Se-SMRP appears limited by poor sensitivity (6).

SMRP is derived from the mesothelin protein (MSLN). MSLN is encoded by the MSLN gene (chromosome 16p13.3) as a precursor protein of $71-\mathrm{kDa}$ that is physiologically cleaved into the $31-\mathrm{kDa}$ megakaryocyte potentiating factor and the $40-\mathrm{kDa}$ mesothelin that is anchored by glycosylphosphatidylinositol to the membrane of mesothelial cells and shed into the biological fluids in which it is referred to as $\operatorname{SMRP}(19,20)$. The production of SMRP may be due to abnormal splicing that results in a secreted form or to cleavage from the membrane by the TNF $\alpha$-converting enzyme ADAM17 (20).

Mesothelin expression was identified in $\sim 90 \%$ of epithelioid MPM and in other cancer types, including lung adenocarcinoma (60\%), and breast (25\%), esophageal $(35 \%)$, ovarian $(60 \%)$, pancreatic, gastric $(50 \%)$ and colon $(40 \%)$ cancer $(19,21)$. Mesothelin expression is more prevalent in aggressive histological subtypes of lung, breast (triple negative) and esophageal (high-grade dysplasia) cancer (21).

Given that the expression of mesothelin is rather limited in numerous normal tissues but elevated in solid tumors, it represents a potential antigen target for therapy in various cancer types, including mesothelioma, pancreatic, breast, colorectal and lung cancer $(5,22)$. In this regard, several therapeutic approaches are under investigation which include the anti-mesothelin immunotoxins SS1P, the chimeric monoclonal antibody amatuximab, the antibody-drug conjugate anetumab ravtansine, the cancer CRS-207 vaccine and chimeric antigen receptors T-cells therapies $(5,22)$.

The prognostic value of Se-SMRP and PE-SMRP levels has also been investigated, but clinical results on this remain uncertain and further studies are required to obtain definitive conclusions (23-34).

The aim of the present study was to highlight the prognostic significance of Se-SMRP in a cohort of Italian patients with MPM. In addition, using a flexible, data-driven model of survival probabilities, the functional form (linear or non-linear) of the dose-response association between Se-SMRP levels and MPM death rates was investigated which, to the best of our knowledge, had not been previously reported in the literature.

\section{Materials and methods}

Patients. The present study included 60 patients with MPM whose sera were collected at diagnosis prior to any treatment in the Pneumology Department of Azienda Sanitaria Locale 5 (ASL 5), La Spezia, Italy, between March 2008 and July 2011. The study period ended in November 2016. Definitive diagnosis was made on the basis of clinical signs, imaging data, cytological examination of PE and tumor biopsy examination staining with hematoxylin-eosin and immunohistochemistry. The characteristics of the patients were collected retrospectively (Table I).

Patient treatment. All patients were treated in the Oncology Department of ASL 5. Thirty-three patients (55.0\%) received first-line therapy, of which $32(53.3 \%)$ had pemetrexed plus cisplatin or carboplatin and $1(1.7 \%)$ had gemcitabine plus carboplatin.

Fifteen patients $(25.0 \%)$ were treated with pemetrexed plus cisplatin in second-line therapy, while $13(21.7 \%)$ had only supportive care and 14 (23.3\%) were treated in other hospitals.

Soluble mesothelin-related protein (SMRP) detection assay. The analysis of SMRP was performed in the Clinical Pathology Department of ASL 5. Aliquots from sera were centrifuged $\left(1,500 \mathrm{x} \mathrm{g}\right.$ for $10 \mathrm{mins}$ at $\left.4^{\circ} \mathrm{C}\right)$ and the supernatant was stored at $-20^{\circ} \mathrm{C}$ until the SMRP analysis was performed.

The determination of Se-SMRP concentrations was performed in duplicate by an ELISA test (ELISA kit 'Mesomark'; cat. no. 801-900; Fujirebio Diagnostic), which has a detection limit of $0.1 \mathrm{ng} / \mathrm{ml}$.

Statistical analyses. Overall survival (OS) time was calculated as the interval between the date of diagnosis and the date of mortality or the last follow-up. Kaplan-Meier analysis was applied to describe and investigate OS probabilities according to tertile-based categories (33rd and 66th percentile) of Se-SMRP and the log-rank test was used to statistically assess differences among OS probabilities. Multivariable Cox regression analysis was performed to estimate death rate ratios (RR) and corresponding $95 \%$ confidence limits (95\% CL). Differences among death rates were assessed using the likelihood ratio test (35). In order to highlight a linear or non-linear dose-response association, potentially overlooked because of the categorization process, a Cox regression model with a restricted cubic spline (RCS) function of Se-SMRP levels was fitted to the OS data. The use of RCS has been widely described as a valid strategy to analyze dose-response associations as it provides greater flexibility for fitting data and modeling more complex associations while adjusting for other covariates (36).

Se-SMRP levels were also log-transformed in order to decrease the influence that outlying measurements may unduly exert on the statistical indexes.

All tests were two tailed and a $\mathrm{P}<0.05$ was considered to indicate a statistically significant difference. All statistical analyses were performed using Stata (StataCorp. Stata Statistical Software; version 14.2).

\section{Results}

Patient characteristics. A total of 60 patients with MPM were studied at diagnosis, prior to any treatment. The patients' mean age was 73 years (range, 60-90 years) and 55 (91.6\%) were 
Table I. Characteristics of patients with malignant pleural mesothelioma analyzed for Se-SMRP.

\begin{tabular}{|c|c|c|}
\hline \multirow{2}{*}{$\begin{array}{l}\text { Patient characteristic } \\
\text { Age, years, at diagnosis, median (range) }\end{array}$} & \multicolumn{2}{|r|}{$\mathrm{n}(\%)$} \\
\hline & \multicolumn{2}{|c|}{$73(60-90)$} \\
\hline \multicolumn{3}{|l|}{ Sex } \\
\hline Male & 55 & (91.6) \\
\hline Female & 5 & $(8.4)$ \\
\hline \multicolumn{3}{|l|}{ Smoking habit } \\
\hline Non-smoker & 28 & $(46.7)$ \\
\hline Current/former smoker & 32 & $(53.3)$ \\
\hline \multicolumn{3}{|l|}{ Asbestos exposure } \\
\hline Unexposed & 21 & $(35.0)$ \\
\hline Exposed & 39 & $(65.0)$ \\
\hline \multicolumn{3}{|l|}{ Symptoms } \\
\hline Dyspnea & 35 & $(58.3)$ \\
\hline Chest pain & 16 & $(26.7)$ \\
\hline Unknown & 9 & $(15.0)$ \\
\hline \multicolumn{3}{|l|}{ IMIG stage } \\
\hline I & 26 & $(43.3)$ \\
\hline II & 18 & $(30.0)$ \\
\hline III/IV & 15 & $(25.0)$ \\
\hline Unknown & 1 & $(1.7)$ \\
\hline \multicolumn{3}{|l|}{ ECOG-PS } \\
\hline 0 & 26 & $(43.3)$ \\
\hline 1 & 22 & $(36.7)$ \\
\hline $2 / 3$ & 12 & $(20.0)$ \\
\hline $\begin{array}{l}\text { Granulocyte/lymphocyte ratio, } \\
\text { median (range) }\end{array}$ & 3.0 & $(0.1-40.1)$ \\
\hline$<2.70$ & 23 & $(38.3)$ \\
\hline $2.70-4.13$ & 16 & $(26.7)$ \\
\hline$>4.13$ & 21 & $(35.0)$ \\
\hline Platelet count, $\mathrm{x} 103 / \mu 1$, median (range) & 285 & $(119-857)$ \\
\hline$<245$ & 18 & $(30.0)$ \\
\hline $245-317$ & 23 & $(38.3)$ \\
\hline$>317$ & 19 & $(31.7)$ \\
\hline \multicolumn{3}{|l|}{ Histological subtype } \\
\hline Epithelioid & 43 & (71.7) \\
\hline Sarcomatoid & 11 & $(18.3)$ \\
\hline Biphasic & 6 & $(10.0)$ \\
\hline \multicolumn{3}{|l|}{ Chemotherapy } \\
\hline Yes & 33 & $(55.0)$ \\
\hline No & 13 & $(21.7)$ \\
\hline Unknown & 14 & $(23.3)$ \\
\hline Se-SMRP, nM, median (range) & 1.24 & $(0.05-13.5)$ \\
\hline$<0.66$ & 17 & $(28.3)$ \\
\hline $0.66-1.46$ & 18 & $(30.0)$ \\
\hline$>1.46$ & 25 & $(41.7)$ \\
\hline Whole sample & & $(100.0)$ \\
\hline
\end{tabular}

IMIG, International Mesothelioma Interest Group staging system adopted by the American Joint Commission on Cancer (AJCC) in the sixth edition of its Cancer Staging Manual and by the International Union Against Cancer (UICC); ECOG-PS, Eastern Cooperative Oncology Group-performance status; Se-SMRP, serum soluble mesothelin-related protein. All data are presented as $\mathrm{n}(\%)$ unless otherwise stated.

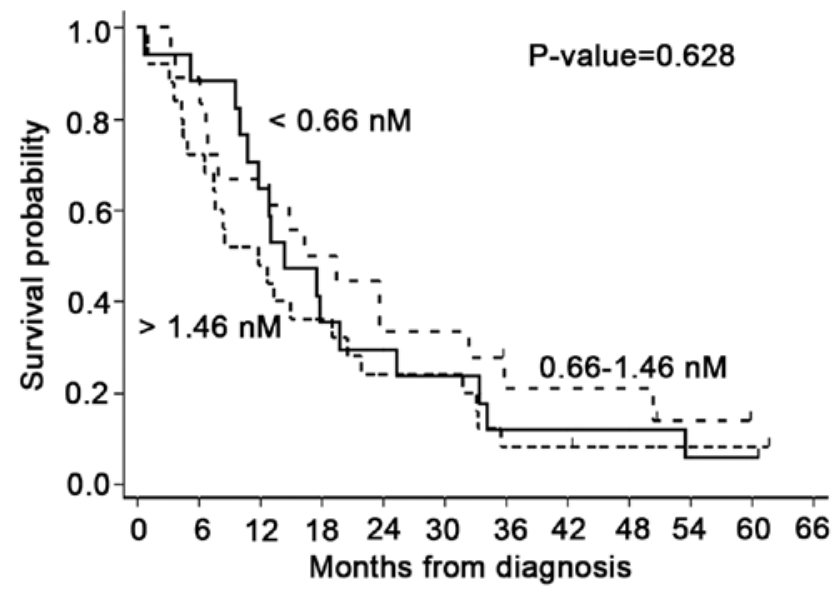

Figure 1. Overall survival probabilities of patients with malignant pleural mesothelioma according to serum soluble mesothelin-related protein levels ( $<0.66 \mathrm{nM}$; 0.66-1.46 nM; >1.46 nM) estimated through the Kaplan-Meier survival analysis.

male. The MPM histology was composed of 43 epithelioid, 11 sarcomatoid and 6 biphasic subtypes. According to the International Mesothelioma Interest Group stage classification, $26(43.3 \%), 18(30.0 \%)$ and $15(25.0 \%)$ patients had MPM at stage I, II and III/IV respectively, while one patient (1.7\%) had a missing value. Twenty-six patients $(43.3 \%)$ had an ECOG-PS score of 0, 22 patients (36.7\%) had an ECOG-PS score of 1 and 12 patients $(20.0 \%)$ had an ECOG-PS score $>1$. Other patient characteristics are summarized in Table I. At the end of the study period, the median follow-up time was 13.9 months (range, 0.7-61.4 months). A total of 54 patients $(90.0 \%)$ died and the median survival time was 13.4 months (95\% CL=10.1-19.4; Table II).

Association between Se-SMRP levels and OS. The baseline median Se-SMRP concentration was $1.24 \mathrm{nM}$ (range, 0.05-13.5 nM; Table I). Kaplan-Meier OS probabilities estimated according to the tertile-based categorization of Se-SMRP levels (<0.66 nM; 0.66-1.46 nM; >1.46 nM) did not show statistically significant differences $(\mathrm{P}=0.628$; Fig. 1$)$. In addition, Cox regression analysis, adjusted for sex, age at diagnosis, disease stage, histological subtype and ECOG-PS, highlighted that patients belonging to the intermediate and higher categories had an RR of 1.87 (95\% CL=0.75-4.67) and 1.95 (95\% CL=0.78-4.92), respectively, compared with patients in the lower category, assumed as reference (Table II). On average, this result corresponds to a death rate increase of $\sim 30 \%$ ( $R R=1.30$; 95\% $\mathrm{CL}=0.73-2.33$ ) per $5 \mathrm{nM}$ unit increase in Se-SMPR (Table II).

Dose-response association between Se-SMRP and mortality rates. In order to identify a dose-response association between Se-SMRP and mortality rates, a multivariable Cox regression model with a RCS function of log-transformed Se-SMRP was used. This method allowed further evidence of a positive association between high Se-SMRP levels and mortality rates to be obtained. In particular, a notably increasing non-linear tendency in mortality rates was estimated when Se-SMRP levels exceeded $1.00 \mathrm{nM}$ (Fig. 2). 
Table II. Effect of Se-SMRP on MPM mortality rates estimated through the Cox regression analysis.

\begin{tabular}{|c|c|c|c|c|c|c|c|c|c|}
\hline \multirow[b]{2}{*}{ Se-SMRP, nM } & \multirow[b]{2}{*}{$\mathrm{N}$} & \multirow[b]{2}{*}{ Mortalities (\%) } & \multicolumn{2}{|c|}{$\begin{array}{l}\text { Follow-up time, } \\
\text { months }\end{array}$} & \multicolumn{2}{|c|}{$\begin{array}{l}\text { Survival time, } \\
\text { months }\end{array}$} & \multirow[b]{2}{*}{ RR } & \multirow[b]{2}{*}{$95 \% \mathrm{CL}$} & \multirow[b]{2}{*}{ P-value } \\
\hline & & & Median & Range & Median & $95 \% \mathrm{CL}$ & & & \\
\hline$<0.66$ & 17 & $16(94.1)$ & 14.4 & $0.7-60.4$ & 14.4 & $10.1-25.3$ & 1.00 & (Ref.) & 0.199 \\
\hline $0.66-1.46$ & 18 & $15(83.3)$ & 17.9 & $3.3-59.6$ & 16.3 & $6.8-32.4$ & 1.87 & $0.75-4.67$ & \\
\hline$>1.46$ & 25 & $23(92.0)$ & 11.8 & $1.0-61.4$ & 11.8 & $6.6-20.5$ & 1.95 & $0.78-4.92$ & \\
\hline Whole sample & 60 & $54(90.0)$ & 13.9 & $0.7-61.4$ & 13.4 & $10.1-19.4$ & $1.30^{\mathrm{a}}$ & $0.73-2.33$ & 0.378 \\
\hline
\end{tabular}

Se-SMRP, soluble mesothelin-related protein in serum; N, patients entered the study; RR, mortality rate ratios adjusted for sex, age at diagnosis, disease stage, histological subtype and ECOG-PS; 95\% CL, 95\% confidence limits for the median survival times and RR; Ref., reference category. ${ }^{a} \mathrm{RR}$ per $5 \mathrm{nM}$ unit increase in Se-SMRP.

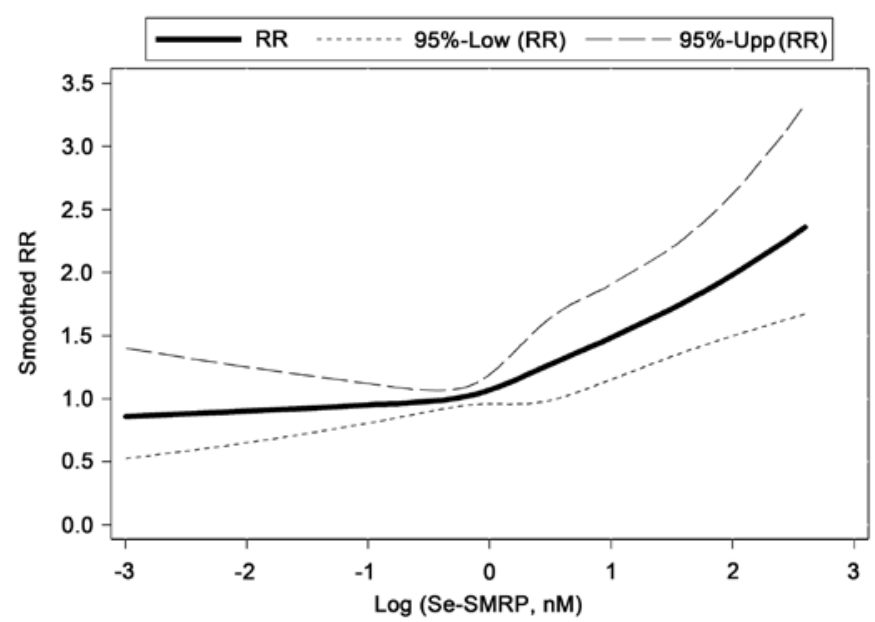

Figure 2. Association between log-transformed Se-SMRP levels and malignant pleural mesothelioma RR estimated through the Cox regression analysis, adjusted for sex, age at diagnosis, disease stage, histological subtype and ECOG-PS, using a three-knot restricted cubic splines. Se-SMRP levels were log-transformed to decrease the influential effect of potential outlying data points. $95 \%$ confidence limits for RR are presented at the top of the figure. 95\%-Low (RR) and 95\%-Upp (RR), 95\% lower and upper limits for RR. RR, mortality risk; Se-SMRP, serum soluble mesothelin-related protein.

\section{Discussion}

The CALGB and EORTC identify ECOG-PS and histological subtypes as the two main prognostic factors of MPM. The ECOG-PS incorporates the patient history and their physical condition to assign a score value according to the deterioration in their health status. However, the ECOG-PS scale is subjective, prone to bias and affected by high inter-observer variability $(2,9)$. By contrast, MPM histological subtypes are carefully defined by a team of pathologists and are considered a more accurate and objective criterion. However, the evaluation of the pathological assessment is performed on tumor material, usually taken by invasive procedures at diagnosis as thoracoscopic biopsies or computed tomography-guided biopsy. These techniques, although tested and efficient, are associated with considerable morbidity and cost, and cannot always be performed due to specific patient conditions $(37,38)$.
Furthermore, the material taken may be inadequate because it is derived from non-tumor areas of the pleura or quantitatively insufficient for histological analysis to be performed.

Additionally, the biphasic subtype may not be diagnosed because the sarcomatoid component, characterized by the morphology of the spindle cells, can be scarce and difficult to be detected $(14,39)$.

Previous studies have focused on identifying novel markers measurable in biological fluids, including serum and PE $(6,13,14)$. Serum is easily obtainable with minimal stress for patients, while PE is present in the majority of MPM patients at diagnosis and routinely withdrawn for diagnosis and therapeutic purposes.

The ideal prognostic biomarker to be determined in serum or PE has not yet been identified. Several biomarkers have been proposed but, to date, none of them can be considered adequate to evaluate the prognosis of MPM because of heterogeneous clinical results in various published studies $(6,13,14)$. By contrast, SMRP may be a prognostic marker for MPM as reported for other malignancies, including ovarian, lung, breast and esophageal cancers $(19,21)$.

A previous study demonstrated that PE-SMRP did not aid in defining the prognosis of MPM. Additionally, the Cox regression modeling, including an RCS had only showed a moderately increasing non-linear trend in the mortality rate. Therefore, it was concluded that PE-SMRP is not recommended for routine use in the clinical management of patients with MPM (40).

In the present study, using the same statistical methodology, the analysis was extended to determine whether Se-SMRP, unlike PE-SMRP, may have a clinical application.

The prognostic value of Se-SMRP is currently highly debated and remains unclear. In a meta-analysis based on results from another study involving 579 patients (18,23-29), Tian et al (30) demonstrated that high levels of Se-SMRP represent a negative, statistically significant prognostic factor for MPM, as assessed in univariate analysis (pooled $\mathrm{RR}=1.96$, 95\% CI: $1.53-2.50, \mathrm{P}<0.001)$, Furthermore, some of these studies demonstrated that the aforementioned association was significant in a multivariable context $(18,23-25)$. 
Pass et al (31), using EDTA plasma, reported that higher levels of plasma SMRP in MPM were associated with a poor prognosis following adjusting for the ECOG-PS, histology, sex, pre-treatment and white blood cell count and suggested SMRP as a possible biomarker to improve the prognostic capability of the EORTC-index (31).

Plasma-SMRP detection is to be considered in the same way as Se-SMRP. Creaney et al (41) reported a significant correlation between the levels of SMRP in serum and plasma (Pearson's coefficient $=0.91 ; \mathrm{P}<0.001$ ).

By contrast, other studies have supported the hypothesis that Se-SMRP has no prognostic significance (18,32-34).

In the present study, using a Cox model including tertile-based categorical terms for Se-SMRP (cut-off points: 0.66 and $1.46 \mathrm{nM}$ ), a positive association between Se-SMRP levels and mortality rates was identified. Such an association was also confirmed using a conservative, flexible and data-driven fitting procedure, namely a RCS function of Se-SMRP, which highlighted a net increase in mortality rates particularly for concentrations of Se-SMRP higher than $1.0 \mathrm{nM}$.

The major limitations of the present study are due to the low statistical power and the high level of patient heterogeneity, with particular reference to the therapeutic treatments. Although such limitations are common to numerous other investigations in this field, it is worth noting that they are two important but distinct issues: The former depends mainly on the small sample size due to the rarity of MPM; the latter on imbalances in the individual baseline characteristics, given the observational (non-experimental) structure of the study design. In the first case, only further and wider investigations, supported by meta-analyses, will be able to confirm the results of the present study from a statistical point of view. In the second case, the use of statistical modeling, including the multivariable Cox regression, is able to mitigate the confounding effect that the aforementioned imbalances can exert on the study association (life expectancy vs. Se-SMRP levels).

In conclusion, the present study provides interesting evidence that Se-SMRP may be considered a prognostic biomarker in patients with MPM. Ultimately, future multicenter studies based on larger cohorts, allowing more powerful statistical analyses, may aid in recognizing Se-SMRP as a prognostic marker useful in routine applications for clinical management of patients with MPM.

\section{Acknowledgements}

The authors of the present study would like to thank Dr E. Battolla and the staff at Laboratory of Clinical Pathology in which the analysis was performed, the staff at the Pneumology Department for collecting serum from patients with mesothelioma and the staff at the Histopathology and Cytopathology Department for performing histological diagnosis at Azienda Sanitaria Locale 5, La Spezia, Italy.

\section{Funding}

The present study was funded by AIL La Spezia-Sezione 'Francesca Lanzone', Project Code MT2019/20, to SR; Italian Ministry of Health, 5x1000 2015, Project Code del.1410/2017 and RC-2019, Project Code M728A, IRCCS Ospedale Policlinico San Martino, to MPP and VF.

\section{Availability of data and materials}

All datasets used and/or analyzed in the present study are available from the corresponding author upon reasonable request.

\section{Authors' contributions}

SR and MPP conceived and designed the study and were responsible for supervising the study. PF, MCF and MT recruited the patients and performed the study. AV, PAC, $\mathrm{UG}, \mathrm{PD}$ and GB were responsible for the management of the patients with mesothelioma. SR, MPP and VF drafted the manuscript. VF and RC performed the statistical analyses. SR, MPP, PD, RC and VF critically revised the manuscript for important intellectual content. All authors have read and approved the final manuscript.

\section{Ethics approval and consent to participate}

The present study was approved by the Liguria Region Ethics Committee (P.R. 207REG2014). All patients enrolled in the study provided written informed consent.

\section{Patient consent for publication}

Not applicable.

\section{Competing interests}

The authors declare that they have no competing interests.

\section{References}

1. Gibbs AR: Role of asbestos and other fibres in the development of diffuse malignant mesothelioma. Thorax 45: 649-654, 1990.

2. Bibby AC, Tsim S, Kanellakis N, Ball H, Talbot DC, Blyth KG, Maskell NA and Psallidas I: Malignant pleural mesothelioma: An update on investigation, diagnosis and treatment. Eur Respir Rev 25: 472-486, 2016.

3. Marinaccio A, Binazzi A, Marzio DD, Scarselli A, Verardo M, Mirabelli D, Gennaro V, Mensi C, Riboldi L, Merler E, et al: Pleural malignant mesothelioma epidemic: Incidence, modalities of asbestos exposure and occupations involved from the Italian national register. Int J Cancer 130: 2146-2154, 2012.

4. Robinson BW: Malignant pleural mesothelioma: An epidemiological perspective. Ann Cardiothorac Surg 1: 491-496, 2012.

5. Yap TA, Aerts JG, Popat S and Fennell DA: Novel insights into mesothelioma biology and implications for therapy. Nat Rev Cancer 17: 475-488, 2017.

6. Pass HI, Alimi M, Carbone M, Yang $\mathrm{H}$ and Goparaju CM: Mesothelioma biomarkers: A review highlighting contributions from the early detection research network. Cancer Epidemiol Biomarkers Prev: Jul 22, 2020 (Epub ahead of print). doi: 10.1158/1055-9965.EPI-20-0083.

7. Billé A, Krug LM, Woo KM, Rusch VW and Zauderer MG: Contemporary analysis of prognostic factors in patients with unresectable malignant pleural mesothelioma. J Thorac Oncol 11: 249-255, 2016.

8. Linton A, Pavlakis N, O'Connell R, Soeberg M, Kao S, Clarke S, Vardy J and van Zandwijk N: Factors associated with survival in a large series of patients with malignant pleural mesothelioma in new south Wales. Br J Cancer 111: 1860-1869, 2014. 
9. Røe OD: Mesothelioma diagnosis and prognosis, are we moving beyond histology and performance status towards circulating biomarkers? J Thorac Dis 10 (Suppl 17): S1956-S1961, 2018.

10. Jean D, Daubriac J, Le Pimpec-Barthes F, Galateau-Salle F and Jaurand MC: Molecular changes in mesothelioma with an impact on prognosis and treatment. Arch Pathol Lab Med 136: 277-293, 2012.

11. Bueno R, Stawiski EW, Goldstein LD, Durinck S, De Rienzo A, Modrusan Z, Gnad F, Nguyen TT, Jaiswal BS, Chirieac LR, et al: Comprehensive genomic analysis of malignant pleural mesothelioma identifies recurrent mutations, gene fusions and splicing alterations. Nat Genet 48: 407-416, 2016.

12. Lo Russo G, Tessari A, Capece M, Galli G, de Braud F, Garassino MC and Palmieri D: MicroRNAs for the diagnosis and management of malignant pleural mesothelioma: A literature review. Front Oncol 8: 650, 2018.

13. Cavallari I, Urso L, Sharova E, Pasello G and Ciminale V: Liquid biopsy in malignant pleural mesothelioma: State of the art, pitfalls, and perspectives. Front Oncol 9: 740, 2019.

14. Arnold DT, De Fonseka D, Hamilton FW, Rahman NM and Maskell NA: Prognostication and monitoring of mesothelioma using biomarkers: A systematic review. Br J Cancer 116: 731-741, 2017.

15. Ferro P, Canessa PA, Battolla E, Dessanti P, Franceschini MC Chiaffi L, Morabito A, Fontana V, Pezzi R, Fedeli F, et al: Mesothelin is more useful in pleural effusion than in serum in the diagnosis of pleural mesothelioma. Anticancer Res 33 2707-2713, 2013.

16. Fontana V, Vigani A, Pistillo MP, Giannoni U, Rosemberg I, Canessa PA, Berisso G, Ferro P, Franceschini M, Tonarelli M and Roncella S: The correlation of serum mesothelin level with pleural thickness in malignant pleural mesothelioma makes it a valuable tool for monitoring tumor progression. J Thorac Oncol 14: e92-e94, 2019.

17. Luo L, Shi HZ, Liang QL, Jiang J, Qin SM and Deng JM: Diagnostic value of soluble mesothelin-related peptides for malignant mesothelioma: A meta-analysis. Respir Med 104: 149-156, 2010

18. Schneider J, Hoffmann H, Dienemann H, Herth FJ, Meister M Muley T and Dienemann H: Diagnostic and prognostic value of soluble mesothelin-related proteins in patients with malignant pleural mesothelioma in comparison with benign asbestosis and lung cancer. J Thorac Oncol 3: 1317-1324, 2008.

19. Chang K and Pastan I: Molecular cloning of mesothelin, a differentiation antigen present on mesothelium, mesotheliomas, and ovarian cancers. Proc Natl Acad Sci USA 93: 136-140, 1996.

20. Lv J and Li P: Mesothelin as a biomarker for targeted therapy. Biomark Res 7: 18, 2019.

21. Morello A, Sadelain M and Adusumilli PS: Mesothelin-targeted CARs: Driving T cells to solid tumors. Cancer Discov 6: 133-146, 2016.

22. Hassan R, Thomas A, Alewine C, Le DT, Jaffee EM and Pastan I: Mesothelin immunotherapy for cancer: Ready for prime time? J Clin Oncol 34: 4171-4179. 2016.

23. Creaney J, Francis RJ, Dick IM, Musk AW, Robinson BW, Byrne MJ and Nowak AK: Serum soluble mesothelin concentrations in malignant pleural mesothelioma: Relationship to tumor volume, clinical stage and changes in tumor burden. Clin Cancer Res 17: 1181-1189, 2011

24. Grigoriu BD, Scherpereel A, Devos P, Chahine B, Letourneux M Lebailly P, Grégoire M, Porte H, Copin MC and Lassalle P: Utility of osteopontin and serum mesothelin in malignant pleural mesothelioma diagnosis and prognosis assessment. Clin Cancer Res 13: 2928-2935, 2007.

25. Cristaudo A, Foddis R, Vivaldi A, Guglielmi G, Dipalma N, Filiberti R, Neri M, Ceppi M, Paganuzzi M, Ivaldi GP, et al: Clinical significance of serum mesothelin in patients with mesothelioma and lung cancer. Clin Cancer Res 13: 5076-5081, 2007.
26. Dipalma N, Luisi V, Di Serio F, Fontana A, Maggiolini P, Licchelli B, Mera E, Bisceglia L, Galise I, Loizzi M, et al: Biomarkers in malignant mesothelioma: Diagnostic and prognostic role of soluble mesothelin-related peptide. Int J Biol Markers 26: 160-165, 2011.

27. Grigoriu BD, Chahine B, Vachani A, Gey T, Conti M, Sterman DH, Marchandise G, Porte H, Albelda SM and Scherpereel A: Kinetics of soluble mesothelin in patients with malignant pleural mesothelioma during treatment. Am J Respir Crit Care Med 179: 950-954, 2009.

28. Creaney J, Yeoman D, Naumoff LK, Hof M, Segal A, Musk AW, De Klerk N, Horick N, Skates SJ and Robinson BW: Soluble mesothelin in effusions: A useful tool for the diagnosis of malignant mesothelioma. Thorax 62: 569-576, 2007.

29. Linch M, Gennatas S, Kazikin S, Iqbal J, Gunapala R, Priest K, Severn J, Norton A, Ayite B, Bhosle J, et al: A serum mesothelin level is a prognostic indicator for patients with malignant mesothelioma in routine clinical practice. BMC Cancer 14: 674, 2014.

30. Tian L, Zeng R, Wang X, Shen C, Lai Y, Wang M and Che G: Prognostic significance of soluble mesothelin in malignant pleural mesothelioma: A meta-analysis. Oncotarget 8: 46425-46435, 2017.

31. Pass HI, Goparaju C, Espin-Garcia O, Donington J, Carbone M, Patel D, Chen Z, Feld R, Cho J, Gadgeel S, et al: Plasma biomarker enrichment of clinical prognostic indices in malignant pleural mesothelioma. J Thorac Oncol 11: 900-909, 2016.

32. Hollevoet K, Nackaerts K, Thas O, Thimpont J, Germonpré P, De Vuyst P, Bosquée L, Legrand C, Kellen E, Kishi Y, et al: The effect of clinical covariates on the diagnostic and prognostic value of soluble mesothelin and megakaryocyte potentiating factor. Chest 141: 477-484, 2012.

33. Ak G, Tada Y, Shimada H, Metintas S, Ito M, Hiroshima K, Tagawa $\mathbf{M}$ and Metintas $\mathbf{M}$ : Midkine is a potential novel marker for malignant mesothelioma with different prognostic and diagnostic values from mesothelin. BMC Cancer 17: 212 , 2017.

34. Creaney J, Dick IM, Meniawy TM, Leong SL, Leon JS, Demelker Y, Segal A, Musk AW, Lee YC, Skates SJ, et al: Comparison of fibulin-3 and mesothelin as markers in malignant mesothelioma. Thorax 69: 895-902, 2014

35. Marubini E and Valsecchi MG: Analyzing Survival Data from Clinical Trials and Observational Studies. John Wiley and Sons, New York, NY, 1995.

36. Harrell FE Jr: Regression modelling strategies. 2nd edition. Springer Verlag, New York, NY, pp22-28, 2015.

37. Walters J and Maskell NA: Biopsy techniques for the diagnosis of mesothelioma. Recent Results Cancer Res 189: 45-55, 2011.

38. Tsao MS, Carbone M, Galateau-Salle F, Moreira AL, Nicholson AG, Roden AC, Adjei AA, Aubry MC, Fennell DA, Gomez D, et al: Pathologic considerations and standardization in mesothelioma clinical trials. J Thorac Oncol 14: 1704-1717, 2019.

39. Galateau Salle F, Le Stang N, Nicholson AG, Pissaloux D, Churg A, Klebe S, Roggli VL, Tazelaar HD, Vignaud JM, Attanoos R, et al: New insights on diagnostic reproducibility of biphasic mesotheliomas: A multi-institutional evaluation by the international mesothelioma panel from the MESOPATH reference center. J Thorac Oncol 13: 1189-1203, 2018.

40. Fontana V, Pistillo MP, Vigani A, Canessa PA, Berisso G, Giannoni U, Ferro P, Franceschini MC, Carosio R, Tonarelli M, et al: Determination of mesothelin levels in pleural effusion does not help predict survival of patients with malignant pleural mesothelioma. Anticancer Res 39: 5219-5223, 2019.

41. Creaney J, Yeoman D, Musk AW, de Klerk N, Skates SJ and Robinson BW: Plasma versus serum levels of osteopontin and mesothelin in patients with malignant mesothelioma-which is best? Lung Cancer 74: 55-60, 2011. 